\title{
$\beta$-Lactamase Production Haemophilus spp. and Resistance to Ampicillin in a General Hospital in Porto Alegre City, RS, Brazil (2001-2005)
}

\author{
Jorge A. S. Ferreira ${ }^{1}$, Andréa Cauduro de Castro' ${ }^{2}$, Marion P. Rocha' ${ }^{2}$, Gustavo Riboldi ${ }^{3}$ and Pedro Alves d'Azevedo ${ }^{2}$ \\ ${ }^{1}$ Department of Microbiology from Hospital Nossa Senhora da Conceição; ${ }^{2}$ Post Graduation Program in Medical Sciences and Clinical \\ Pathology from Fundação Faculdade Federal de Ciências Médicas de Porto Alegre (FFFCMPA); ${ }^{3}$ Post Graduation Program in Molecular \\ Biology from Biotechnology Center, Federal University of Rio Grande do Sul (UFRGS); Porto Alegre, RS, Brazil
}

\begin{abstract}
In a four-year period (July/2001-June/2005), 410 Haemophilus spp. isolates were studied. Those were isolated from sputum at Hospital Nossa Senhora da Conceição (NSC) in Porto Alegre city (RS). $\beta$-lactamase enzyme was detected in $113(27.6 \%)$ of isolates through chromogenic cephalosporin method. Fifty-eight $(51.3 \%)$ of them showed sensibility to ampicillin through disc-diffusion method using Haemophilus Test Medium (HTM) by NCCLS criteria. In 297 $(72.4 \%)$ isolates $\beta$-lactamase was not detected by chromogenic cephalosporin method. Five $(1.7 \%)$ of them were resistant and $1(0.3 \%)$ intermediate to ampicillin using disc-diffusion method. The authors emphasized the importance of Haemophilus spp. resistance to ampicillin research in clinical laboratories routine and the use of more than one method for this analysis was proposed, due to different resistance mechanisms in Haemophilus spp.

Key-Words: Haemophilus spp., $\beta$-lactamase, antimicrobial resistance, ampicillin.
\end{abstract}

In the recent years, the resistance to antimicrobials among pathogens implied in respiratory tract infection (RTI) acquired in community have increased and spread in alarming rates [1]. Haemophilus influenzae is an important pathogen in RTI acquired in community, causing signs and symptoms undistinguished from those caused by other pathogens, notoriously Streptococcus pneumoniae [2]. Haemophilus influenzae is a Gram-negative pathogen and important etiologic agent of morbid-mortality among children under five years old, both in developed countries and in Latin American countries. Among these, $H$. influenzae type B (Hib-capsulated) is considered one of the most important etiologic agent of fatal infections in children [3], whereas encapsulated $H$. influenza is part of superior respiratory tract flora, and it can be considered a protection shield against invasive infections [4]. Since the 1970's, there has been an worldwide increase of $H$. influenzae resistant isolates, mainly to ampicillin. The ampicillin-resistance is mediated by plasmids through transposons (TnA - bacteria mobile chromosome elements) and this mechanism is extremely important to plasmids evolution originating multiple resistance [5]. The resistance of $H$. influenzae to ampicillin is not widely known and discussed [6]. The production of $\beta$-lactamase is the main mechanism of ampicillin-resistance and other beta-lactamics [7]. Between 1972 and 1974 the first resistance to ampicillin and chloranphenicol $H$. influenzae was isolated in the United States and Europe, with consequent increasing and diffusion of those strains worldwide [8-10]. The resistance of $H$. influenzae to ampicillin has been described, characterizing $\beta$ lactamase extracellular TEM-1 [11], and ROB-1 [12], both

Received on 17 June 2006; revised 13 December 2006.

Address for correspondence: Dr. Andréa Cauduro de Castro. Departamento de Microbiologia da FFFCMPA. Rua Sarmento Leite 245/211 - Porto Alegre - RS - Brazil. Zip code: 90050-170. E-mail: candrea@ghc.com.br.

The Brazilian Journal of Infectious Diseases 2007;11(1):50-52. (C) 2007 by The Brazilian Journal of Infectious Diseases and Contexto Publishing. All rights reserved. acquired through plasmids. Recently another type of ampicillin resistance has been described, mediated by elements other than $\beta$-lactamase, once a phenotipic BLNAR ( $\beta$-lactamase negative ampicillin resistant) characteristic has been shown?[13]. Such resistance has been associated to the presence of altered PBPs (penicillin binding proteins) [14]. The detection of $\beta$-lactamase in clinical microbiology laboratories shows a controversial scenary due to the presence of many methods and variable proposes [6,15]. The most acceptable methods are the ones recommended by National Committee for Clinical Laboratory Standards (NCCLS) [16]. The purpose of this study was to describe the consecutive analysis of 410 strains of Haemophilus ssp. isolated from sputum between July/2001 and June/2005 and show the importance of the application of more than one analyzes method in the analysis of antimicrobial resistance.

\section{Material and Methods}

Hospital

The microbiology department of Hospital Nossa Senhora da Conceição (HNSC), in Porto Alegre, RS, Brazil.

\section{Isolates}

Within the period of July 2001 to June 2005, 410 Haemophilus spp. were isolated from sputum in the HNSC and were prospectively submitted to resistance tests to antimicrobials. The isolates which were obtained in the period between January and September 2004 have been excluded due to non-commercial availability of the $\beta$-lactamase test through chromogenic cephalosporin test, mentioned below.

Isolation

Four hundred and ten Haemophilus spp. strains isolated from sputum in chocolate-agar (BioMérieux, Jacarepaguá, Rio de Janeiro) have been tested in microaerophilic condition; increasing condition with low tension production of $\mathrm{CO}_{2}$ (3 to $5 \%$ of $\mathrm{CO}_{2}$ for Haemophilus spp.) by the mean of a candle put inside an appropriate jar. 
Suscetibility Testing

The chromogenic cephalosporin test, also known as nitrocefin (cefinase test) (BD BBL-Becton Dickinson and Company, Sparks, USA) has been applied $[17,18]$. The cefinase discs contain nitrocephin (chromogenic cephalosporin), and each disc is used to evaluate the production of $\beta$-lactamase in a clinic isolated. When the production of this enzyme occurs with the action of bacteria, the discs (with yellowish initial color) are shown in a reddish color in the respective area where the isolated $\beta$ lactamase producer was. The second test was the detection of ampicillin resistance using the disc diffusion method, adapted from Kirby-Bauer method to application for $H$. influenzae according to NCCLS (2001 to 2005) recommendations. Haemophilus Test Medium (HTM) was used from Oxoid (Basinstoke, Hampshire, England) and discs of $10 \mathrm{mg}$ of ampicillin from Cefar (Cefar Diagnóstica LTDA, São Paulo, SP, Brazil).

\section{Results}

Four hundred and ten strains of Haemophilus spp. isolated in HNSC from July 2001 to June 2005 were submitted to suscetibility test to antimicrobials through different methods, with phenotipic characteristics according to data shown in Table 1 . All the strains and tests were made prospectively. The strains have been consecutively included in the study, except during the period from January to September 2004, when there was not commercial availability for the $\beta$-lactamase detection method. Table 1 shows the decreasing of incidences of Haemophilus spp. $\beta$-lactamase-positive and ampicillinresistance isolates (BLPAR) during the period of study until 2004. In 2005 the emerge of $\beta$-lactamase-negative and ampicillin-resistant (BLNAR) takes place, with following incidence of $\beta$-lactamase -negative and ampicillin intermediate (BLNAI) and BLNAR. Table 2 shows that out of the 410 Haemophilus spp., 14.1\% are BLPAS and signs the emerge of 5 BLNAR cases, which means about $1 \%$ of the total isolated ones and $10 \%$ of the total ampicillin-resistant (AR), which becomes an alarming fact in the institution.

\section{Discussion}

Antibiotic self-administration is a common practice in Latin American countries, which contributes for the increase of many resistant organisms, including the Haemophilus spp. ampicillin resistance. This might occur due to the antibiotic administration by patients with respiratory tract infection providing a selective pressure and decrease of suscetibility to $\beta$-lactamase [5]. Moreover, the different protocols of recommendation for use of antibiotics in such situation take to a selective pressure in the community, which is observed in different resistant levels in Brazil and other countries [19]. In countries like Brazil, there is some need of better analysis standard for Haemophilus spp. resistance in order to assure a better therapy option, mainly where the ampicillin and chloranfenicol combination is used in large scale (taking into account their low cost and effectiveness) [20]. The data shown from the evaluation of results obtained in the ampicillin suscetibility test versus $\beta$ lactamase test in the cefinase test, present the decrease in the incidence of isolates of positive Haemophilus spp. $\beta$ lactamase and ampicillin resistance from 2002 on, and the negative $\beta$-lactamase ampicillin resistance from 2003 on with some increase in 2005. The importance of the cefinase test is shown in Table 2, where we can see 14\% of BLPAS that should induct to ampicillin administration if the cefinase test was not done. Apart from that, the use of isolated cefinase test, in the last 2 years, would not detect those BLNAR strains that correspond to $10 \%$ of all the ampicillin resistant. Taking into account all the data shown, the authors reinforce the importance of the use of the cefinase test in clinical microbiology laboratories as a complementary test for Haemophilus spp. resistant studies.

Table 1. Distribution of 410 Haemophilus spp. isolates from sputum at Hospital Nossa Senhora da Conceição, Porto Alegre (RS)

\begin{tabular}{lccccccc}
\hline Years & BLP/AS & BLP/AI & BLP/AR & BLN/AS & BLN/AI & BLN/AR & Total \\
\hline 2001 & 26 & 4 & 20 & 62 & 0 & 0 & 102 \\
2002 & 12 & 2 & 11 & 75 & 0 & 0 & 100 \\
2003 & 8 & 0 & 3 & 84 & 0 & 1 & 96 \\
2004 & 5 & 1 & 1 & 29 & 1 & 0 & 37 \\
2005 & 7 & 2 & 11 & 41 & 0 & 4 & 65 \\
\hline
\end{tabular}

The data represent the number of Haemophilus spp. by period. BLP= $\beta$-lactamase positive; BLN= $\beta$-lactamase negative; $\mathrm{AS}=$ ampicillin suscetible; $\mathrm{AI}=$ ampicillin intermediate; $\mathrm{AR}=$ ampicillin resistant.

Table 2. Behavior of 410 Haemophilus spp. strains in the $\beta$-lactamase and ampicillin disc test

\begin{tabular}{lrcrc}
\hline & \multicolumn{1}{c}{ AS } & AI & \multicolumn{1}{c}{ AR } & Total \\
\hline BLP & $58(14.1 \%)$ & $9(2.2 \%)$ & $46(11.2 \%)$ & $113(27.6 \%)$ \\
BLN & $291(71.0 \%)$ & $1(0.24 \%)$ & $5(1.21 \%)$ & $297(72.4 \%)$ \\
Total & $349(85.1 \%)$ & $10(2.4 \%)$ & $51(12.4 \%)$ & \\
\hline
\end{tabular}

The data represent the number of Haemophilus spp., where the numbers in parentheses represent the percentage corresponding. $\mathrm{BLP}=\mathrm{b}$-lactamase-positive; $\mathrm{BLN}=\mathrm{b}$-lactamasenegative; AS=ampicillin-suscetible; $\mathrm{AI}=$ ampicillin-intermediate; $\mathrm{AR}=$ ampicillin-resistant. 


\section{References}

1. Felmingham D. Envolving resistence patterns in communityacquired respiratory tract pathogens: first results from the PROTEKT global surveillance study. Prospective Resistant Organism Tracking and Epidemiology for the Ketolide Telithromycin Infect 2002;44:33-10.

2. Nicholson S.C., Webb C.D., Andriole V.T., et al. Haemophilus influenzae in respiratory tract infections in community - based clinical practice: therapy with gatifloxacin. Diagn Microbiol Infect Dis 2002;44:101-7.

3. Peltola H. Worlwide Haemophilus Influenzae type B disease at the beginning of the 21 century: global analysis of the disease burden 25 years after the use of the polysaccharide vaccine and a decade after the advent of conjugates. Clin Microbiol Rev 2000; $13: 302-17$.

4. Giebink G.S. The microbiology of otitis media. Pediatr Inf Dis 1989;8:S18-S20.

5. James P.A., Lewis D.A., Jordens J.Z., et al. The incidence and epidemiology of ß-lactam resistance in Haemophilus influenzae. J Antimicrob Chemother 1996;37:737-46.

6. Dias C.A.G. Resistência de Haemophilus influenzae à ampicilina: uma revisão. Revista AMRIGS 1995;39:157-61.

7. Hoban D., Felmingham D. The PROTEKT surveillance study: antimicrobial susceptibility of Haemophilus influenzae and Moraxella catarrhalis from community - acquired respiratory tract infections. J Antimicrob Chemother 2002;50:49-59.

8. Jacobs M.R., et al. Susceptibilities of Streptococcus pneumoniae and Haemophilus influenzae to 10 oral antimicrobial agents based on pharmacodynamics parameters: 1997 US Surveillance study. Antimicrob Agents Chemoter 1999;43:1901-8.

9. Jordens J.Z., Slack M.P. Haemophilus influenzae: then and now. Eur J Clin Microbiol Infec Dis 1995;14:935-48.

10. Jorgensen J.H. Update on mechanisms and prevalence of antimicrobial resistance in Haemophilus influenzae. Clin Infec Dis 1992; $14: 1119-23$.
11. Bijlmer H.A., van Alphen L., Greenwood B.M., et al. Antibiotic susceptibility of invasive and non-invasive isolates of Haemophilus influenzae from the Gambia, West Africa. J Antimicrob Chemother 1994;34:275-80.

12. Markowitz S.M. Isolation of ampicillin resistant production strain of Haemophils influenzae. Antimicrob Agents and Chemother 1980; $17: 80-3$.

13. Doern G.V. Antimicrobial resistance among lower respiratory tract isolates of Haemophilus influenzae: results of a 1992-93 Western Europe and USA collaborative surveillance study. J Antimicrob Chemother 1996;38:59-69.

14. Ubukata K., Chiba N., Hasewaga K. Differentiation of $\beta$-lactamasenegative ampicillin-resistant Haemophilus influenzae from other $H$. influenzae strains by a disc method. Journal of Infection and Chemotherapy 2002;8:50-8.

15. Barry A.L., Fuchs P.C., Brown S.D. Identification of BetaLactamase- Negative, Ampicillin-Resistant Strains of Haemophilus influenzae with Four Methods and Eight Media. Antimicrob Agents Chemother 2001;45:1585-8.

16. Nacional Comittee for Clinical Laboratory Standards (NCCLS), Clinical and Laboratory Standards Institute, USA, ed. 2001, 2002, 2003, 2004 and 2005.

17. Montgomery K.L., Raymundo J., Drew W.L. Chromogenic cephalosporin spot test to detect beta-lactamase in clinically significant bacteria. J Clin Microbiol 1979;9:205-7.

18. Lee D.T., Rosenblatt J.E. A comparison of four methods for detecting beta-lactamase in anaerobic bacteria. Diagn Microbial Infect Dis 1983; Jun1(2):173-5.

19. de Andrade A.L.S.S., Brandileone M.C., Di Fabio J.L., et al. Haemophilus influenzae resistance in Latin America: systematic review of surveillance data. Microb Drug Resist 2001;7(4):40311.

20. Hussey G., Hitchcock J., Coetzee G., et al. Serotypes and antimicrobial susceptibility of Haemophilus influenzae. J Antimicrob Chemother 1994;34(6):1031-6. 